\title{
Deploying a community mental health team for the effective care of individuals with schizophrenia
}

\section{Geraldine Strathdee}

Community mental health teams (CMHTs) have their origin in the American Community Mental Health Centre programme of the 1960s. The teams were mandated to provide services with an emphasis on the needs of the severely mentally ill (SMI). However, over time the focus of work became 'the worried well', to the neglect of those with severe mental illness. CMHTs are now a firm feature of British psychiatry. ASainsbury Centre study recently identified over three hundred CMHTs across England, defining themselves as comprising at least four members, from two or more disciplines, with a caseload of individuals with mental health disorders, largely residing in the community.

In 1994, the focus of work, organisation, approach to service development and styles of management of British teams were the subject of intensive study by a number of national agencies. All the reviews comment on the variability of targeting of the severely mentally ill by the CMHTs. The Audit Commission and Mental Health Foundation Reports in particular make recommendations for achieving more focused and effective team development.

This article is based on the experience of clinicians involved in the planning and development of two CMHTs in urban areas which focused on the SMI and incorporates some of the main recommendations of the national reviews.

\section{A strategic approach}

A strategic approach to the planning and implementation of services and working practices is essential if a CMHT is to be effective in treating the severely mentally ill, including those with schizophrenia. The strategy should be informed by an understanding of the theoretical and research bases of community care programmes and, equally important, a knowledge of where this has been translated into good practice, not just in well funded 'model' programmes but also in pragmatic working practices in the UK (Test \& Scott, 1990).

Box 1 outlines the key elements which such a strategy should include. First, recognition of the range of needs of patients with schizophrenia. Second, the establishment of the components of a system of service structures between the disciplines and agencies involved in the care of the mentally ill. Third, the use of a range of effective therapeutic interventions which address the wider needs of the severely mentally ill and which have been demonstrated to result in improved health, social and other outcomes. Fourth, the use of explicit and effective organisation and management techniques within the CMHT and the service system.

Finally, it should include an analysis of the current and needed skill mix of the team members, a plan for continuing development through training, and establishment of mechanisms of support for staff.

\section{Understanding the needs of people with schizophrenia}

The development of community services has had two consistent themes: that the traditional inherited service systems dominated by large institutions should be replaced with a more balanced and flexible range of alternative services, and that

Geraldine Strathdee, MRCPsych, is a consultant psychiatrist in Rehabilitation and Community care at the Bethlem and Maudsley NHS Trust, and a senior medical officer on an 18-month secondment to the Mental Health Policy Unit, Department of Health. She is the consultant member of PACT, a local sector case management and assertive outreach team for those with severe mental illness, and has been involved in the development of community mental health services in Greenwich as a member of a generic CMHT. 
Box 1. Strategy for the effective deployment of CMHTs

Recognition of the needs of individuals with SMI

Establishment of the components of a comprehensive community mental health service

Use of effective interventions

Effective organisation and management of the CHMT

Planning of staff skill mix, training and support

services should be directed to meeting individual needs. Individuals with severe and the more common mental health disorders have a wide range of social and health needs. The needs of both groups are similar in nature and may differ only in degree. They include housing, welfare benefits, physical and dental care, medication, psychological treatments, work and rehabilitation (Strathdee \& Sutherby, 1995).

\section{The components of a comprehensive community mental health service system}

One of the most positive aspects of British psychiatry in the past five years has been the emergence of a consensus of the components of a comprehensive community mental health service system (Box 2). These have been articulated by policy makers (Department of Health, 1993), clinicians (Royal College of Psychiatrists, 1995), and by user groups such as MIND (1983). The role of CMHTs in assessing the need for, and planning and development of some of these components will be discussed in detail below.

\section{Epidemiology}

To plan strategically a community team must first assess the full extent of the service needed. The starting point for any CMHT must be to develop, over a one to three year timetable, a detailed knowledge of the number and needs of individuals with schizophrenia and other SMI in their area. By using epidemiological techniques teams can estimate the expected number of individuals with schizophrenia for their area. The OPCS National Morbidity survey has produced surprising findings of wider variation in the prevalence of psychosis in England than has previously been identified. This varies from $0.2 \%$ in affluent areas to $0.9 \%$ in more deprived areas.

Of those with psychiatric morbidity, it is likely that only a proportion will be in contact with the specialist services. Between $25-40 \%$ may be in contact only with their GP, social services, the housing agencies, police, local churches or user groups. A fundamental building block in establishing an effective team is to develop an identification component of the service, developing regular liaison with all other agencies who may be serving the needs of the severely mentally ill in the community. Box 3 indicates the likely contact points of people with schizophrenia. In many services nationally the implementation of the Care Programme Approach (CPA) has been used as the vehicle to audit how the number of SMI in contact with the service compares with that expected from the epidemiological data.

\section{Needs assessment for rational service planning}

Having identified individuals with schizophrenia in the sector, the next step is the introduction of a system of needs assessment. The routine collection of a clinical data set and care planning assessment and review is vital, and when incorporated into the CMHT culture has the following effects: it

Box 2. Components of a comprehensive mental health service

Identification and needs assessment systems

Care Programme Approach integrated with care management

Hospital and Community beds and bed management

Case management and assertive outreach

Day care, rehabilitation, education and work opportunities

Crisis response

Assessment and consultation

Carer and community education and support

Primary care liaison

User advocacy and community alliances 
ensures that services are developed based on the aggregated needs of patients; informs the case for resources; facilitates multi-agency coordination of services planning and delivery; and is vital at a national level for the mental health agenda (Kingdon, 1994).

Despite the many standardised assessment instruments in mental health, remarkably few are used in routine clinical practice. This discrepancy may be because although many instruments are helpful in monitoring symptoms, they do not lead directly to informed decision making or management plans. In general, any innovation which is seen as a time-consuming addition to, rather than an integral part of, routine clinical practice is unlikely to succeed. Newer instruments are a first stage in combining research validity with practical application. These include the Health of the Nation Outcome Scales and the Camberwell Assessment of Need.

\section{Case management and assertive outreach services}

To meet the complex needs of people with schizophrenia, case management as a service or at least a technique is essential in drawing together all the necessary services into one coherent package of care. In practice, case management for the longterm mentally ill has developed into a range of techniques that can be described along 12 different axes (Thornicroft, 1990), which aim to ensure that patients with long-term psychiatric disorders receive consistent and continuing services for as long as they are required. Studies have compared a number of models of case management which offer acute home-based care with hospital care (Stein \& Test, 1980; Hoult et al, 1984; Muijen et al, 1992).

Despite differences in the models and evaluative methodologies used, these and several other studies confirm a decrease in hospital admissions, improvement in clinical outcomes and social functioning and greater patient satisfaction from acute homeorientated care. However CMHTs cannot regard case management as a panacea for success in treating people with schizophrenia. It is only effective where those practising case management employ effective intervention techniques and where it is supported by an adequate infrastructure of resources.

\section{Hospital E community beds}

People with schizophrenia account for $60 \%$ of all in-patient bed use in mental health units. It is essential that a CMHT's use of hospital beds, the most expensive resource, is necessary and based on sound therapeutic reasons. A great deal of controversy has surrounded the number, use and management of acute mental health beds. The evidence points to the complexity of the problems and the need to develop a broad range of services to resolve them. Some services have required additional acute beds to deliver safe care (Department of Health, 1994). Lelliott \& Wing's (1994) study of new long-stay patients found that $61 \%$ were considered to be inappropriately placed in hospital beds, $47 \%$ required a community based residential setting, and of these over half were in hospital because no suitable community placement was available. Several analyses of acute bed unit use indicate that: $30-70 \%$ of those in the beds are 'revolving door regulars', well known to services; and that a significant proportion had been readmitted within three months of discharge, due

Box 3. Identification of patients with schizophrenia

1 Mental health service contacts Out-patient and domiciliary visit records CPA, Supervision register and S.117 records CPN case-load and depot clinic patients Mental Health Act and in-patient audit data Crisis attenders e.g. A\&E attenders Residents of long-stay institutions

2 Primary care team contacts

Practice register diagnosis of psychosis

Repeat psychotropic drug prescriptions Frequent emergency and other consultations Hostel/group home/sheltered residence populations

CPN attenders and health visitor contacts

3 Social service contacts

Area social worker case-loads

Care management recipients

Housing department clients causing concern

4 Voluntary sector and other agency contacts Residents of sheltered accommodation

Individuals presenting to churches in distress

Individuals causing local beat officers concern

Imprisoned and homeless people

Probation officer case-loads

Drop-in and other casual facility users

User groups 
to a lack of provision of high support community accommodation and effective discharge planning mechanisms.

The success of community based services is crucially related to the nature and availability of accommodation with appropriate levels of support. CMHTs must have a wide range of hospital and community beds available to them. These should range from responsive and community-sensitive secure facilities for mentally disordered offenders, through to acute hospital beds, 24-hour staffed units in hospital hostels and community places for the new long stay, especially those with mild to moderate challenging behaviours, and a range of community beds for crisis diversion, quarter- and half-way hostel purposes, respite facilities, and residential and permanent accommodation. CMHTs are ideally placed to play a leadership role in ensuring the planning of such facilities if they have concrete local data to present on the numbers of their patients with schizophrenia and the number and range of bed provision needed.

\section{CMHTs and bed management}

In addition to planning and ensuring the development of an adequate number and range of beds, community teams have been shown to be effective in preventing unnecessary and inappropriate acute bed use by a range of techniques. Organisation of their service on a defined sector basis facilitates comprehensive case identification, needs assessment and effective inter-agency planning. In a study where the CMHTs' senior and experienced clinicians did initial home-based assessments rather than on the hospital out-patient clinic site, admission was reduced by $50-70 \%$ (Burns et al, 1993). Gate-keeping, with senior doctors and nurses becoming involved in any decision to admit a patient, can support junior staff, who are less experienced in assessment of risk and safe alternative to admission.

As research has repeatedly shown continuity of care is important. The most likely time of readmission and suicide attempts is in the first 46 weeks after discharge. Follow-up by keyworkers is more likely to be successful if it is intense in this vulnerable period. Another of the most consistent research findings is that integrated hospital and community services are essential to maximise the use of resources. Unless community teams have control over their own hospital beds, both bed use and length of stay are significantly increased.

\section{Crisis response services}

Individuals with schizophrenia are vulnerable to environmental stressors which may result in exacerbation of symptoms and relapse, and episodes of acute illness. Successful CMHTs must aim to provide support and help for the client, family members, and others to cope with the emergency while maintaining the client's status as a functioning community member to the greatest extent. Studies of users, carers and GPs produce almost inter-changeable findings. All want crisis services to be available at a single point of telephone or face-to-face contact. They would value services on a 24-hour, 7-day basis, manned by experienced and known mental health professionals and providing crisis diversion facilities as a viable alternative to hospitalisation. Nationally this type of service is provided by a limited number of teams. Even when the severely mentally ill are in contact with CMHTs, it is often the GP or casualty staff with limited training who provide the majority of crisis care.

\section{Day care and rehabilitation programmes}

In a comprehensive review, Holloway (1988) defines three possible functions of the day hospital for the severely mentally ill: as an alternative to hospital admission when acutely ill; for provision of support, supervision and monitoring in the transition between hospital and home; as a source of longterm structure and support for those with chronic handicaps; a site for brief intensive therapy for those who require short-term focused rehabilitation; and an information, training and communication resource. Wood (1994) as the occupational therapist responsible for the development of day care services for a CMHT stresses the importance of user consultation and the formation of formal liaison with other local providers of work, education and day care.

\section{Assessment and consultation services}

Until the past few decades the majority of consultation services were conducted in hospital out-patient settings. Evaluation of these services indicates dissatisfaction with communication patterns, clinical and referral outcomes and up to $45 \%$ non-attendance. The response of clinicians, uninformed by policy or research considerations, 
Box 4. Primary care liaison in the care of individuals with schizophrenia

Communication strategy

Provide directory of street boundaries, sector team names, roles and contact numbers

Information booklets of therapies available and named contacts to advise on appropriate referrals

Crisis services

Single and well publicised telephone contact access point

Agreed crisis response patterns

Assessment and out-patient services Agreed referral-appointment interval Stated referral criteria for members of the CMHT

Clear communication with stated objectives of management, predicted response, complications and side-effects

Six-monthly review plans for longer-term patients

Clearly stated role of GP and specialist in treatment

Clarification of prescribing responsibilities

Shared care for people with schizophrenia Set up a joint case register similar to that for diabetes

Support CPN focus on SMI

Agree annual physical care reviews

Ensure GP has verbal/physical/fax input into CPA care planning reviews

Identify GP/practice nurse role in development of relapse prevention plan and crisis contracts

Agree protocols for group homes/hostels Discuss consequences of expensive new psychotropic drugs and ECRs with total fund-holders

Obtain support for appropriate range of beds and community alternatives to hospital admission

was to work on a sessional basis in primary care centres. Early criticisms of the clinics was that they would result in psychiatrists serving only the worried well. The evidence refutes this. They provide a service to groups of the SMI who previously dropped out of contact with the specialist services i.e. women with long-term disorders, young men with schizophrenia, the homeless with psychoses, and those with paranoid disorders who felt stigmatised by attendance at a hospital site (Tyrer, 1984).

In many community services community psychiatric nurses were the first to develop attachments to particular general practices. Where the nurses have been hospital based and work as members of the secondary care team, $80 \%$ of their referrals are from psychiatrists and individuals with SMI form a considerable proportion of their workload. In other organisations where nurses, although employed by secondary care services, are attached to particular general practices, the referral pattern shifts with $80 \%$ of their referrals coming direct from GPs. Caseloads tend to be large, composed of patients with neurotic and adjustment disorders.

Although the caseloads of both the hospital based and primary care based CPNs remain similar in terms of the numbers of individuals with schizophrenia, the mean time in contact with psychotic patients is a third of the time spent with nonpsychotic patients and is almost entirely limited to the administration of injections.

\section{Primary care liaison}

One of the great strengths of the British health care system is its primary care infrastructure. To a large extent its significant involvement with people with schizophrenia goes unrecognised. Although the numbers of such patients are small (about 4-7 per GP with an average list size of around 2000), their use of primary care services is high where they are not well supported by the CMHT. The move to GP purchasing power through fund-holding requires teams to become advocates of the SMI.

Based on research findings in the primary care area and the implementation of a successful shared care model for diabetes and asthma (also longerterm conditions). Box 4 proposes a range of initiatives to improve joint working between the primary health care teams and the CMHT.

\section{Effective interventions for schizophrenia}

However well a CMHT has developed the service structures, outcomes for their patients with schizophrenia will only improve where there is an analysis of the skills of the team, funding dedicated for training in acquiring new skills, and the routine use of effective interventions (Conway et al, 1994). Box 5 is not comprehensive but outlines some of the most important. 
Box 5. Effective interventions for people with schizophrenia

Housing with adequate support

Welfare benefits and financial advice

Physical and dental care

Medication and psycho-educational programmes

Behavioural-cognitive therapy for psychotic symptoms

Identification and development of coping strategies

Crisis prevention and relapse prevention

Family problem solving therapy

Rehabilitation, practical skills and support

Work and education

\section{Psychosocial interventions}

In a recent comprehensive review Slade et al (1995) found that up to $45 \%$ of the severely mentally ill do not apply for, or receive their entitlement to, welfare benefits. The provision of welfare benefits to provide an adequate standard of living is a major priority for service users. Motivation to attend day care programmes or out-patient clinics is likely to increase if a bus fare to attend rather than buying food no longer holds. Likewise having permanent, affordable, comfortable housing is important in achieving a stable mental state free of worries about rent, damp, harassing neighbours and so forth. Craig's (1995) studies of the homeless have shown that outcomes are improved when care programmes include interventions which address housing needs.

\section{Physical health and dental care}

Co-existing physical morbidity is common in patients with longer-term schizophrenia. In a study of long-term day centre attenders, Brugha et al (1989) found that $41 \%$ suffered medical problems potentially requiring care. Annual physical review as a minimum should be arranged with the GP by the patient's keyworker.

\section{Medication and psycho- educational programmes}

Medication is an important element in the management of many people with schizophrenia in the acute and longer-term phases of their illness. It is important that members of community teams familiarise themselves with good practices in medication prescribing (Thompson, 1994). An understanding of the need for regular review, monitoring of side-effects, tailoring medication type and dosage to individual needs, both in the acute and continuing care phases, is basic good practice. Where easily accessible psycho-educational packages of information on conditions and treatments (preferably developed jointly with users and carers) are used, compliance with treatment is enhanced and relapse prevention increased.

\section{Family and carer education, support and therapies}

Adequate early treatment, associated with client, family, and staff education and training, can prevent the onset of many crises (Birchwood et al, 1989). Falloon et al (1993) showed reductions in family disruption, physical and mental disorders, and perceived burden after structured family interventions. Because of the episodic nature of the illness, however, there will be instances that require acute care and rapid response crisis stabilisation services. The need to see the individual in the context of his or her family environment is well understood. Six controlled studies have illustrated the impact of wider intervention packages on relapse rates in households with high EE. Successful programmes contain the following features: a problem-focused approach to reduce expressed emotion and stress in the home environment; an educational component; long-term application for six to nine months; involvement of the patient in the family treatment; well trained intervention staff; and close liaison with members of the clinical team and other agencies (Kuipers \& Bebbington, 1991).

\section{Effective organisation of CMHTs}

The effective care of patients with schizophrenia requires the skills of nurses, occupational therapists, psychologists, social workers and psychiatrists. No one discipline working autonomously has the range of skills required if clinical and social outcomes are to be improved. Therefore there are a number of key organisational questions for CMHTs to consider. First, how can the team develop, not as a set of independent and auto- 
Box 6. Issues to consider in developing an operational policy for management of a CMHT

Team aims and client groups served

Catchment area and local needs assessment

Team functions

Referrals to and from the team: criteria for acceptance and discharge

Team membership

Team relationships and accountability mechanisms

Team meetings: conduct agendas decision-making procedures

Case allocation and management

Team members' role: all members key workers team manager/co-ordinator

Systems and procedures: healthcare records (format, access and confidentiality)

budget formulation and monitoring, evaluation of service \& of individual members

Staff development, support and training

Details of service provided

Team plan (6-month, one-year and three-year plans with costs)

Appendices: reports of workload, services provided, grants obtained

nomous practitioners, but with multi-disciplinary and preferably, inter-agency integrated working. Both the Audit Commission and the Mental Health Taskforce London studies found that the development of community service which targeted the SMI was most advanced in those areas where there were integrated health and social CMHTs with clear management structures.

Second, how should the team organise to protect the SMI. In the majority of services CMHTs are generic i.e. they provide care to both new referrals, those requiring acute care and also those with SMI. Unless effective management systems are in place to ensure a continuing prioritisation of the most needy and vulnerable, the drift towards treating the less ill is well described. Services have developed a number of organisational formats to prevent this trend.

In some areas a separate rehabilitation service exists where a dedicated case management and assertive outreach team serves a limited number of those with SMI across several sectors. Within generic sector teams there are a number of strategies. Agreed case-loads/case-mix are regularly monitored to ensure that the proportion of those with SMI remains high. An additional strategy is the appointment of specialist case managers or rehabilitation experts within the generic team. For them to develop or retain specialist expertise they have dual membership of both their generic sector team and a district-wide rehabilitation service. In areas where the prevalence of SMI is high, sectors have divided their total pool of staff resource to form two sector teams, one providing acute care while the other provides case management and specialist rehabilitation services which emphasise normalisation and community integration.

Whatever the arrangement, case-loads must be realistic, with one case manager for ten patients requiring assertive outreach and high levels of input. The ration extends to $1: 30-40$ where the individuals in the caseload have had comprehensive assessments, well developed care plans, are stable, engaged and trusting of services offered, rehearsed relapse prevention and crisis contracts, and well supported by a range of community agencies.

\section{Management of CMHT}

The development of an operational policy is a prerequisite for the effective working of any CMHT. Box 6 serves as a useful basis for both team building and service development.

In British psychiatry we have a consensus about the range of services we need to offer, the interventions we recognise as effective and the outcomes we hope to achieve. Effective organisation and management of CMHTs is an essential building block to the future. There is no standard blueprint to guide individuals teams. Success depends on a shared vision at local level, alliances forged between community agencies and solutions developed based on local strengths and resources. As Tansella concludes "Only the past can be copied, the future must be created".

\section{Acknowledgements}

I am grateful to Maurice Perry, Helen Wood, Ann Watts and the members of my PACT community mental health team in Peckham for their inspiration and help in the preparation of this paper. I particularly acknowledge Graham Thornicroft for his contribution to our joint ventures in the planning and development of services for individuals with severe mental illness at the Maudsley hospital which have informed many of the views expressed in this paper. 


\section{References}

Birchwood, M., Smith, J., MacMillan, F., et al (1989) Predicting relapse in schizophrenia: the development and implementation of an early signs monitoring system using patients and families as observers. Psychological Medicine, 19, 649-656.

Brugha, T, Wing, J. \& Smith, B. (1989) Physical ill-health of the long-term mentally ill in the community. Is there an unmet need? British Journal of Psychiatry, 155, 777-782.

Burns, T., Deadsmore, A., Bhat, A., et al (1993) A controlled trial of home based acute psychiatric services. British Journal of Psychiatry, 163, 49-61.

Conway, M., Melzer, D., Shepherd, G.,et al (1994) A Companion to Purchasing Adult Mental Health Services. London: Sainsbury Centre for Mental Health.

Department of Health (1993) The Health of the Nation: Key Area Handbook. Mental Illness. London: HMSO.

- (1994) Mental Health in London: Priorities for Action. Mental Health Task Force London.

Falloon, I., LaPorta, M., Fadden, G., et al (1993) Managing Stress in Families. Cognitive and Behavioural Strategies for Enhancing Coping Skills. London: Routledge.

Holloway, F. (1988) Day care and community support. In Community Care in Practice (eds A. Lavender \& F. Holloway). Chichester: Wiley.

Hoult, J., Rosen, A. \& Reynolds, I. (1984) Community orientated treatment compared to psychiatric hospital orientated treatment. Social Sciences and Medicine, 18, 1005-1010.

Kingdon, D. (1994). Making care programming work. Advances in Psychiatric Treatment, 1, 41-46.

Kuipers, L. \& Bebbington, P. (1991) Working in Partnership: Clinicians and Carers in the Management of Long Term Illness. London: Heinemann.

Lelliott, P. \& Wing, J. (1994). A national audit of new long-stay psychiatric patients II. Impact on services. British Journal of Psychiatry, 165, 170-178.

MIND (1983) Common Concerns. London: MIND Publications.

Muijen, M., Marks, I., Connolly, J. et al (1992) Home-based care and standard hospital care for patients with severe mental illness: a randomised controlled trial. British Medical Journal, 304, 749-754.

Slade, M., McCrone, P. \& Thornicroft, G. (1995) Uptake of welfare benefits by psychiatric patients. Psychiatric Bulletin, 19, 411413.

Stein, L. \& Test, M. (1980) Alternative to mental hospital treatment. I. Conceptual model, treatment programme and clinical evaluation. Archives of General Psychiatry, 37, 392-397.

Strathdee, G. \& Jenkins, R. (1995) Contracting for primary care mental health services. In Purchasing Mental Health Services (eds G. Thornicroft \& G. Strathdee). London: HMSO.

- \& Sutherby, K. (1995) Liaison psychiatry and primary health care settings. In Multi-Professional Cooperation in Community Care (eds S. Ritter, N. Watkins, N. Hervey, et al). London: Edward Arnold.

Tansella, M. (1991) Community care without mental hospitals ten years experience. Psychological Medicine, Suppl. 19, 4748.

Tes, T. M. \& Scott, R. (1990) Theoretical and research bases of community care programmes. In Mental Health Care Delivery: Innovations, Impediments \& Implementation (eds I. Marks \& R. Scott). Cambridge: Cambridge University Press.

Thompson, C. (1994) Consensus statement. The use of high-dose anti-psychotic medication. British Journal of Psychiatry, 164, 448458.
Thornicroft, G. (1990) Case management for the severely mentally ill. Social Psychiatry and Psychiatric Epidemiology, 25, 141-143.

Tyrer, P. (1984) Psychiatric clinics in general practice: an extension of community care. British Journal of Psychiatry, 145, 571-575.

Wood, H. (1994) Planning day services. In Planning and Developing Community Mental Health Services (eds K. Thomson \& G. Strathdee). London: PACT, Maudsley NHS Trust.

\section{Multiple choice questions}

1 An operational policy for a CMHT should contain details of
a priority client group
b referral criteria
c case allocation
d staff training plan
e interventions offered.

2 Effective deployment of a CMHT requires

a a data base of the severely ill population to be served

b at least 20 staff

c a devolved budget to keyworkers

d only acute hospital beds

e staff trained in effective interventions.

\section{CMHTs}

a should follow a service development blue print which takes no account of local needs

b can improve outcomes of patients with schizophrenia by ignoring housing needs

c are usually in contact with all the local SMI

d work best as autonomous disciplines, not a team approach

e should use case management for individuals with schizophrenia.

\begin{tabular}{|ccc|c|}
\hline \multicolumn{3}{|c|}{ MCQ answers } \\
\hline 1 & 2 & 3 \\
a T & a T & a F \\
b T & b F & b F \\
c T & c F & c F \\
d T & d F & d F \\
e T & e T & e T \\
\hline
\end{tabular}

